\title{
VII.
}

\section{Untersuchungen aus dem Laboratorium der medicinischen Klinik zu Königsberg i. Pr.}

\author{
Mitgetheilt von \\ Dr. O. Minkowski, \\ Privatdocent und Assistent an der medieinischen Klinik zu Königsberg i. Pr. \\ I. Ueber das Verhalten des Muskelglykogens nach der \\ Leberexstirpation von M. Laves. ${ }^{1}$ )
}

Die Frage, ob der Muskel selbständig Glykogen bilden könne, hat zuerst $\mathrm{K} \ddot{u} \mathrm{lz}^{2}$ ) durch Ausschaltung der Leber experimentell zu entscheiden versucht. Nach dem Vorgange von J. Müller, Kunde und Moleschott exstirpirte er bei Fröschen die Leber und constatirte, dass auch nach der Entleberung eine Steigerung des Glykogengebalts in den Muskeln zu Stande kam, wenn den Thieren Lösungen von Traubenzucker unter die Hant gespritzt wurden. Damit schien es bewiesen, dass der Muskel auch unabhängig von der Leber Glykogen bilden könne.

Die Differenzen, welche $\mathrm{K} \ddot{\mathrm{I}} \mathrm{z} z$ in dem Glykogengehalte der Muskeln bei den verschiedenen Versuchen gefunden hatte, waren indessen sehr gering. Auch konnte vielleicht die Beweiskraft dieser geringen Differenzen noch dadurch beeinträchtigt erscheinen, dass die vergleichenden Bestimmungen an verschiedenen Thieren ausgeführt waren, und zwar an Winterfröschen, welche, wie bekannt, einen äusserst trägen Stoffwechsel zeigen.

Herr Laves benutzte daher die im Laboratorium der Königsberger medicinischen Klinik bereits vielfach geübte Methode der Leberexstirpation bei Vögeln, um die Frage nach dem Ursprung des Muskelglykogens einer erneuten Prüfung zu unterziehen. Er operirte an Hühnern und Gänsen, denen er zunächst unmittelbar nach der Leberexstirpation ein Stïck eines Pectoralmuskels entnahm, um dessen Glykogengehalt zu bestimmen. Einige Zeit später (nach 1-13 Stunden) wurden die Thiere durch Nackenstich getödtet, sofort ein Stïck des zweiten Pectoralmuskels excidirt und auch in diesem der Glykogengehalt bestimmt. $\mathrm{Zu}$ diesen Glykogenbestimmungen bediente

1) Inaug.-Dissertation. Königsberg 1886.

2) Bildet der Muskel selbständig Glykogen? Pflüger's Arch. 24. Bd. S. 64. 1881. 
sich Herr Laves des von $K \ddot{u} 1 \mathrm{z}^{1}$ ) nach genauen kritischen Untersuchungen zweckmässig modificirten Brücke'schen Verfahrens.

Es ergab sich nun, dass der längere Zeit nach der Leberexstirpation untersuchte Pectoralmuskel stets erheblich weniger Glykogen enthielt, als der im Beginne des Versuches exstirpirte Muskel.

Folgende Tabelle giebt die bei den einzelnen Versuchen erhaltenen Resultate wieder:

TABelle I.

\begin{tabular}{|c|c|c|c|c|}
\hline $\mathrm{Nr}$. & Thierart & $\begin{array}{l}\text { Zeitintervall } \\
\text { zwischen beiden } \\
\text { Glykogen- } \\
\text { bestimmungen }\end{array}$ & $\begin{array}{l}\text { Glykogengehalt } \\
\text { des ersten Muskels } \\
\text { in Proe. }\end{array}$ & $\begin{array}{c}\text { Glykogengehalt } \\
\text { des zweiten Muskels } \\
\text { in Proc. }\end{array}$ \\
\hline $\begin{array}{l}1 \\
2 \\
3 \\
4 \\
5 \\
6 \\
7 \\
8 \\
9\end{array}$ & $\begin{array}{l}\text { Huhn } \\
= \\
= \\
= \\
\text { Gans } \\
= \\
= \\
= \\
=\end{array}$ & $\begin{array}{cl}11 / 2 \text { Stunden } & \\
31 / 2 & = \\
4 & = \\
13 & = \\
1 & = \\
3^{1 / 2} & = \\
4^{1 / 2} & = \\
7^{1 / 2} & = \\
9 & =\end{array}$ & $\begin{array}{l}0,431 \\
0,528 \\
0,477 \\
0,791 \\
0,377 \\
0,520 \\
0,344 \\
0,699 \\
0,544\end{array}$ & $\begin{array}{l}0,238 \\
0,147 \\
0,144 \\
0,261 \\
0,119 \\
0,100 \\
0,037 \\
0,120 \\
0,100\end{array}$ \\
\hline
\end{tabular}

In allen in dieser Tabelle aufgeführten Versuchen waren die Thiere vor der Entleberung reichlich mit Gerste und Hafer gefüttert worden. Nach der Operation nahmen sie keine feste Nahrung mehr zu sich. Dieser letztere Umstand konnte indessen nicht als Ursache für den rapiden Schwund des Muskelglykogens betrachtet werden, da, wie schon $W$ eiss ${ }^{2}$ ) und Luchsinger ${ }^{3}$ ) gezeigt haben, das Muskelglykogen sich im Hungerzastande sehr langsam vermindert. Dementsprechend fand Laves auch in zwei Versuchen nach dreiund fünftägiger Carenz in der Pectoralmusculatur noch 0,205 und 0,328 Proc. Glykogen. Auch hier sank nach Ausschaltung der Leber der Glykogengehalt sehr rasch weiter, so dass er nach $3 \frac{1}{2}$ bezw. 9 Stunden nur noch 0,175 und 0,108 Proc. betrug. Uebrigens kam eine Abnahme des Glykogengehaites in den Muskeln in nicht geringerem Grade zu Stande, wenn den Thieren nach der Entleberung noch 20-30 g Traubenzucker in wiederholten kleinen Dosen mittelst Schlundsonde in den Magen gebracht wurden, wie aus folgender Tabelle ersichtlich ist:

1) Zeitschrift f. Biologie. XXII. Bd. S. 161.

2) Sitziungsber. d. Wiener Akademie. LXVII. Bd. 1873.

3) Experimentelle und kritische Beiträge zur Physiologie und Pathologie des Glykogens. Inaug,-Dissertation. Zürich 1875. 
Untersuchungen aus dem Laboratorium der med. Klinik zu Königsberg i. Pr. 141

TABeLLE II.

\begin{tabular}{|c|c|c|c|c|c|}
\hline Nr. & Tbierart & $\begin{array}{c}\text { Zeitintervall } \\
\text { zwisehen beiden } \\
\text { Glykogen- } \\
\text { bestimmungen }\end{array}$ & $\begin{array}{l}\text { Glykogengehalt } \\
\text { ersten Muskels } \\
\text { in Proc. }\end{array}$ & $\begin{array}{c}\text { Glykogengehalt } \\
\text { des } \\
\text { zweiten Muskels } \\
\text { in Proc. }\end{array}$ & $\begin{array}{l}\text { Eingegebene } \\
\text { Menge } \\
\text { Traubenumcker } \\
\text { in } g\end{array}$ \\
\hline 10 & Gans & $3^{1 / 2}$ Stunden & 0.312 & 0,072 & 30 \\
\hline 11 & Huhn & $9=$ & 0,328 & 0,108 & 20 \\
\hline 12 & $=$ & 10 & 0,600 & 0,189 & 20 \\
\hline 13 & $=$ & 12 & 0,856 & 0,109 & 30 \\
\hline
\end{tabular}

Dass der eingeführte Tranbenzucker auch resorbirt wurde, konnte in diesen Versuchen dadurch gezeigt werden, dass bei dem Tode der Thiere noch Zucker im Blute nachweisbar war, während soust, wie Laves in Uebereinstimmung mit den Angaben des Referenten ${ }^{1}$ ) gefunden hat, nach der Leberexstirpation der Zucker aus dem Blute sehr bald vollständig geschwunden war.

Dass es in der That die Ausschaltung der Leberfunction und nicht. etwa blos der operative Eingriff als solcher war, welcher das Schwinden des Muskelglykogens zur Folge hatte, konnte durch verschiedene Controlversuche bewiesen werden. Zunächst zeigte es sich, dass die Exstirpation des einen Pectoralis selbst nach 24 Stunden noch keine merkliche Aenderung in dem Glykogengehalte des anderen Pectoralmuskels bewirkte. Um nun zu prüfen, welchen Einfluss grössere, mit Eröff́nung der Abdominalhöble verbundene Operationen auf den Glykogengehalt der Muskeln ausüben, resecirte Laves grössere Stücke des Dünndarms bis zu einer Ausdehnung von $50 \mathrm{~cm}$ Länge. Auch nach dieser sehr eingreifenden Operation fanden sich keine nennenswerthen Differenzen im Glykogengehalte, wie Tabelle III zeigt:

TABeLle III.

\begin{tabular}{|c|c|c|c|c|}
\hline $\mathrm{Nr}$. & Thierart & $\begin{array}{l}\text { Zeitintervall } \\
\text { zwischen beiden } \\
\text { Glykogen- } \\
\text { bestimmungen }\end{array}$ & $\begin{array}{l}\text { Gilykogengebalt d. vor } \\
\text { dex Darmresection } \\
\text { exstirpirten Muskels } \\
\text { in Proc. }\end{array}$ & $\begin{array}{l}\text { Glykogengeh. d. nach } \\
\text { der Darmresection } \\
\text { exstirpirten Muskels } \\
\text { in Proo. }\end{array}$ \\
\hline $\begin{array}{l}14 \\
15 \\
16\end{array}$ & $\begin{array}{c}\text { Huhn } \\
= \\
=\end{array}$ & $\begin{array}{l}7 \text { Stunden } \\
8 \stackrel{=}{9}=\end{array}$ & $\begin{array}{l}0,415 \\
0,434 \\
0,038\end{array}$ & $\begin{array}{l}0,386 \\
0,311 \\
0,005\end{array}$ \\
\hline
\end{tabular}

In einigen anderen Fällen wurảe bei Hühnern der Muskelmagen exstirpirt und hierbei zeigte es sich, dass nach dieser Operation eine nicht unbedeutende Abnahme des Glykogens in den Muskeln zu Stande kam. Doch ist zu berücksichtigen, dass die Exstirpation des Magens, infolge der eigenthümlichen Gefässanordnung, sehr erhebliche Circulationsstörungen in der Leber zur Folge haben muss.

1) Dieses Archiv. XXI, Bd. S. 74. 
Demnach ist man berechtigt, die rasche Verminderung des Muskelglykogens nach der Leberexstirpation auf das Ausfallen der Leberfunction zu beziehen. Es dürfte diese Verminderung des Glykogens dadurch zu erklären sein, dass der Glykogenvorrath des Muskels rascher aufgebraucht wird, wenn die Hauptquelle der Glykogenbildung in der Leber versiegt. Da ferner auch nach reichlicher Zufuhr von Traubenzucker eine Abnahme des Muskelglykogens zu verzeichnen war, so erscheint es unwahrscheinlich, dass der Muskel selbständig - wenigstens aus Traubenzucker - Glykogen zu bilden vermag.

\section{Ueber experimentelle Glykosurie bei Vögeln von A. Thiel.1)}

Den Untersuchungen von Thiel lag ursprünglich die Absicht zu Grunde, die Methode der Leberexstirpation bei Vögeln dazu zu benutzen, um über die Bedeutung der Leber für das Zustandekommen der experimentellen Glykosurien Aufschluss zu erhalten. Bei den meisten experimentellen Glykosurien glaubt man allgemein, die Ausscheidung des Zuckers im Harne als die Folge einer Functionsstörung der Leber betrachten zu dürfen, und zwar, wie viele Autoren annehmen, als die Folge einer übermåssigen Production von Zucker in der Leber. Ob aber für alle Glykosurien und speciell auch für den Diabetes mellitus beim Menschen eine solche Erklärung zulässig ist, erscheint mindestens zweifelhaft. Es war daher von vorneherein nicht unwahrscheinlich, dạss unter den verschiedenen experimentellen Glykosurien sich solche unterscheiden lassen werden, welche nur bei erhaltener Leber zu Stande kommen, und solche, welche auch nach Exstirpation der Leber erzengt werden können.

Die Versuche von Thiel ergaben nun zunächst in dieser. Hinsicht ein negatives Resultat, insofern als die meisten Eingriffe, durch welche bei Säugethieren künstliche Glykosurien erzeugt werden können, bei den Vögeln in der Regel versagten und nur in ganz vereinzelten Fällen ein Auftreten sehr geringer Mengen von Zucker im Harne zur Folge hatten.

Eine einzige Ausnahme macht das von v. Mering ${ }^{2}$ ) angegebene Phloridzin. Nach Eingabe von $3-5 \mathrm{~g}$ dieser Substanz konnte bei Enten constant eine ziemlich erhebliche Glykosurie beobachtet werden, welche 5-8 Stunden anhielt. In maximo wurden in einem

1) Inang.-Dissertation. Königsberg 1887.

2) Ueber experimentellen Diabetes. Verhandl, des V. Congresses für innere Medicin.. Wiesbaden 1886. S. 185. 
Versuche innerhalb 5 Stunden $200 \mathrm{ccm}$ Harn mit 3,5 g Zucker erhalten. Auf das Zustandekommen dieser Glykosurie war - wie es v. Mering gleichfalls schon angegeben hatte - die vorausgegangene Exstirpation der Leber von keinem merklichen Einflusse.

Zum Nachweis des Zackers wurde in diesen, wie in allen übrigen Versuchen, der Harn nach Unterbindung des Darms oberhalb der Cloake rein aufgefangen, bei mässiger Wärme auf dem Wasserbade eingeengt, mit heissem Weingeist extrahirt, der alkoholische Auszug nach dem Erkalten abfiltrirt und eingedampft, der Rückstand in Wasser gelöst und eventuell noch mit Bleizueker behandelt. In dem entbleiten Filtrate wurde dann der Zucker durch die Trommersche und die Gährungsprobe nachgewiesen.

Ausser der Eingabe von Phloridzin wurden noch folgende Methoden zur Erzeugung einer Glykosurie versucht:

1. Der Zuckerstich. Claude Bernard ${ }^{1}$ ) hatte bereits den Zuckerstich bei Tauben mit negativem Resultate ausgeführt. Im Gegensatze dazu hat M. Bernhardt2) in den Excrementen von Tauben, welche er längere Zeit mit Fleiseh gefüttert hatte, um den Darminhalt zuckerfrei zu machen, nach der Piquâre Zucker nachweisen können. Allerdings liess sich die Glykosurie in diesen Versuchen nicht so leicht demonstriren, wie bei dem Piquûre-Diabetes der Kaninchen: es mussten erst die geringen Mengen der während mehrerer Tage. entleerten und ïberallhin im Käfig verschmierten Excremente zusammengekratzt und verarbeitet werden, um das Material für die Zuckerproben zu liefern. - Thiel stellte, wie bereits erwähnt, seine Versuche in der Weise an, dass stets vor Ausführung der Piquûre der Darm oberhalb der Cloake unterbunden wurde und auf diese Weise der Harn rein, ohne Beimengung von Darminhalt aufgefangen werden konnte. In 9 derartigen Versuchen an Kühen, bei welchen der Zuckerstich zum Theil nach Eröffnung des Ligamentum atlanto-occipitale ansgeführt war, konnte im Harn auch nicht eine Spur von Zucker nachgewiesen werden.

2. Vergiftung mit Kohlenoxyd und Leuchtgas. Trotzdem die Thiere $6-8 \mathrm{mal}$ hintereinander bis zum Eintreten von Asphyxie vergiftet wurden, traten nur in einem einzigen Falle ganz vorübergehend zweifelhafte Spuren von Zucker auf, in vier anderen Versuchen war Zncker im Harn überhaupt nicht nachweisbar.

1) Leçons sur la physiologie et la pathologie du système nerveux. Paris 1858. Vol. I. p. 461.

2) Ueber d. Zuckerstich bei Vögeln. Virchow's Archiv. 59. Bd. S. 407. 1874. 
3. Amylnitrit. Im Anschluss an die oben erwähnten Untersuchungen über den Zuckerstich hatte Bernhardt auch einige Versuche mit Amylnitrit angestellt, ohne aber zu einer definitiven Beantwortung der Frage zu 'gelangen, ob das Amylnitrit auch bei Vögeln, wie A. Hoffmann ${ }^{1}$ ) es für das Kaninchen nachgewiesen hatte, Auftreten von. Zucker im Harn bewirkt. Thiel machte an mebreren Hühnern subcutane Injectionen ron Amylnitrit und fand danach niemals Zucker im Harn, trotzdem die Dosis von $0,2-0,3$ allmählich bis zu tödtlichen Dosen $(2,0-3,0 \mathrm{~g})$ gesteigert wurde.

4. Orthonitrophenylpropiolsäure (Georg Hoppe-Seyl er $\left.{ }^{2}\right)$ ). Diese Säure wurde zum Theil in Substanz; zum Theil als Natronsalz gelöst in kleinen $(0,3)$ und grösseren $(2,0-3,0 \mathrm{~g})$ Dosen per os and subcutan eingeführt. Es trat Blaufärbung des Harns (Indigo) auf, aber unter 12 Versuchen konnte nur ein einziges Mal nach Eingabe von $2 \mathrm{~g}$ der Säure Zucker im Harn nachgewiesen werden, und zwar: auch nur in einer Menge von 0,05 g. - Ein Controlversuch an einem Hunde ergab eine zwei Tage anhaltende ziemlich reichliche Zuckerausscheidung.

5. Milchsäure $\left(G_{0} \operatorname{ltz}^{3}\right)$ ). Nach Einführung grösserer Mengen $(5-8 \mathrm{~g})$ von Milchsäure in den Magen starben die Thiere häufig am folgenden Tage; eine Glykosurie wurde aber niemals beobachtet.

6. Curarevergiftung. Nach $4-6$ stündiger tiefer Curarenarkose bei künstlicher Respiration traten Spuren von reducirender Substanz im Alkoholextracte des Harns auf. Die Gährungsprobe ergab ein negatives Resultat.

Die Absicht, in welcher diese Untersuchungen begonnen waren, musste bald aufgegeben werden, in Anbetracht der Schwierigkeiten, welche die Erzeugung einer künstlichen Glykosurie bei den Vögeln darbot. Wie ist nun aber dieses eigenthümliche Verhalten des Vogelorganismus zu erklären?

Man dürfte wohl nicht fehl gehen, wenn man die Schwierigkeit, bei Vögeln eine Glykosurie zu erzengen, mit der ausserordentlich intensiven Oxydationsthätigkeit im Organismus dieser Thiere in $\mathrm{Zu}$ sammenhang bringt. Eine Steigerung der Zuckerproduction kann beim Vogel nicht so leicht zu einem Auftreten von Zucker im Harne

1) Archiv für Anatomie und Physiologie. 1873. S. 746.

2) Zeitschrift für physiologische Chemie. VII. Bd. 1883.

3) Wirkungen der Milchsäure auf den thierischen Organismus. Inaug.-Diss. Berlin 1868. 
Veranlassung werden, weil sein Organismus sich leichter durch gesteigerte Oxydation des Kohlenbydratüberschusses zu entledigen vermag, als derjenige des Sängethieres.

Dass das Phloridzin auch bei Vögeln, und zwar selbst nach Exstirpation der Leber nach Glykosurie erzeugt, spricht dafür, dass das Zustandekommen der Zuckerausscheidung hier anf andere Momente zurückzuführen ist, als bei den übrigen bis jetzt untersuchten experimentellen Glykosurien. Wahrscheinlich handelt es sich beim Phloridzin, wie v. Mering angenommen hat, nicht um eine gesteigerte Zuckerproduction, sondern um eine Beeinträchtigung des Zuckerverbrauchs im Organismus oder um eine Einwirkung auf die secretorische Thätigkeit der Nieren.

\section{Ueber den Eisengehalt der Galle bei Polycholie von 0 . Baserin. ${ }^{1}$ )}

Bei der Umwandlung des Blutfarbstoffs in Gallenfarbstoff findet in der Leber zunächst eine Abspaltung von Eisen aus dem Hämatin statt. Das abgespaltene Eisen kann bei der Polycholie, welche nach Arsenwasserstoff- und Toluilendiaminvergiftung zu Stande kommt, wie Naunyn und Minkowski2) gezeigt haben, in der Leber nachgewiesen werden, und zwar findet sich hier das Eisen einmal in blutkörperchenhaltigen Zellen innerhalb der Lebercapillaren und zweitens in den Leberzellen selbst. In diesen letzteren liegen die die Eisenreaction gebenden Körnchen meistens in dem Theile der Zelle, welcher der Gallencapillare zugekehrt ist, was besonders an den Lebern von Vögeln, die mit Arsenwasserstoff vergiftet wurden, zu erkennen war. Diese eigenthtimliche Anordnung der Eisenkörnchen längs der Gallencapillaren legte die Vermuthung nahe, dass das bei der Zerlegung des Blutfarbstoffs in der Leber abgespaltene Eisen durch die Galle ausgeschieden werde. Herr Baserin suchte nun durch directe Bestimmungen des Eisengehalts der Galle zu entscheiden, ob diese Vermuthung richtig ist.

$\mathrm{Zu}$ diesem Zwecke wurden bei Gallenfistelhunden einige Eisenbestimmungen in der normalen Galle gemacht, darauf die Thiere mit Arsenwasserstoff vergiftet und in der alsdann entleerten, sehr farbstoffreichen Galle ebenfalls der Eisengehalt bestimmt. Zur quantitativen Bestimmung des Eisens wurde die Galle veraseht und in der Asche das Eisen in der üblichen Weise nach Reduction mittelst schwefliger Säure mit übermangansaurem Kali titrirt.

1) Die ausführlichere Mittheilung dieser Untersuchungen soll in einer Inaug.Dissertation erfolgen.

2) Dieses Archiv. XXI. Bd. S. 24. 
Da es zunächst nur darauf ankam, zu entscheiden, ob überhaupt eine Steigerung des Eisengehalts in der Galle gleichzeitig mit der Zunahme des Gallenfarbstoffs zu Stande kommt, so wurden genauere quantitative Bestimmungen des Gallenfarbstoffs nicht ausgeführt, sondern eine Vermehrung desselben nur aus der stärkeren Verdünnung der Galle erschlossen, welche nothwendig war, um eine bestimmte Farbennuance (z. B. Nr. 3 der Vogel'schen Harnfarbenscala) zu erzielen. Selbstrerständlich konnte dieses Verfahren nur eine höchst oberflächliche Schätzung gestatten; die Differenzen in dem Farbstoffgehalte der Galle vor und nach der Arsenwasserstoffvergiftung waren aber bei einzelnen Versuchen so gross, dass Fehlerquellen überhaupt nicht in Betracht kamen. 1) Um eine gleiche Farbennuançe zu erzielen, musste bei mehreren Versuchen die nach der Vergiftung erhaltene Galle auf das 7-16 fache der Gallenmenge verdünnt werden, welche vor der Vergiftung in den gleichen Tageszeiten gesammelt worden war.

Im Gegensatz zu dieser sehr erheblichen Vermehrung des Gallenfarbstoffs zeigte es sich nun, dass der Eisengehalt keine merklichen Veränderungen erlitten hatte. Vor der Vergiftung schwankte derselbe je nach Grösse des Versuchsthieres zwischen 1 bis $3 \mathrm{mg}$ in 8 Stunden; in der nach der Vergiftung secernirten Galle waren annähernd ebenso grosse, bei einzelnen Versuchen sogar noch etwas geringere Eisenmengen nachweisbar.

Um zu entscheiden, ob die vermehrte Eisenausscheidung nicht etwa der vermehrten Farbstoffansscheidung nachfolge, wurden in einzelnen Versuchen, in welchen die Thiere die Vergiftung ïberstanden hatten, die Eisenbestimmungen noch 14 Tage lang fortgesetzt; aber auch von einer nachträglichen Vermehrung des Eisengehaltes war in diesen Versuchen nichts nachweisbar.

Ueber den Eisengehalt der Hundegalle hat $\mathrm{Kunkel}{ }^{2)}$ zuerst genauere Untersuchungen angestellt. Er fand in der Fistelgalle durchschnittlich $0,006 \mathrm{~g}$ Eisen in $100 \mathrm{ccm}$ Galle und berechnete aus einer Vergleichung mit der ausgeschiedenen Bilirubinmenge, dass nur etwa

1) Der Einwand, dass die dunklere Farbe der Galle möglicherweise nicht auf eine Vermehrung des Bilirubins, sondern auf eine Beimengung anderer Farbstoffe bezogen werden müsste - ein Einwand, der übrigens in gleicher Weise auch bei den complicirteren spectroskopischen Methoden der Bilirubinbestimmung gemacht werden könnte - ist dadurch zurückzuweisen, dass die Farbennuance bei genügender Verdünnung stets die gleiche war, wenn nur die Galle möglichst frisch untersucht wurde.

2) Eisen- und Farbstoffausscheidung in der Galle. Pflüger's Archiv. 14. Bd. S. 353.1877 . 
der siebente Theil des bei der Umwandlung des Hämatins in Bilirubin frei werdenden Eisens in der Galle enthalten sei. Er nahm daher an, dass bei dem Zerfalle des Hämatins ein eisenreicher Rest abgespalten und grösstentheils zurtickgehalten werde.

Wie aus den Untersuchungen von Baserin nun hervorgeht, steht die Menge des in der Galle ausgeschiedenen Eisens nicht im Verhältniss zu der Menge des Gallenfarbstoffes, sondern es hängt offenbar von anderen noch unbekannten Momenten ab, ein wie grosser Bruchtheil des aus dem Hämatin abgespaltenen Eisens in der Galle zur Ausscheidung gelangt.

Die besondere Anordnung der eisenhaltigen Körnchen in den Leberzellen, wie sie Naunyn und Minkowski beschrieben haben, kann demnach nicht mit der Eisenausscheidung durch die Galle in Zusammenhang gebracht werden. Dadurch gewinnt die von $\mathrm{Nau}$ ny n und Minkowski gemachte Annahme, dass das in den Leberzellen nachweisbare Eisen anch in der Leberzelle selbst abgespalten ist, dass somit auch die Bildung des Gallenfarbstoffs, wenigstens zum Theil in der Leberzelle selbst von Statten geht, noch weiter an Wahrscheinlichkeit. ${ }^{1}$ )

Was mit dem in der Leber zurïckbleibenden Eisenreste - der übrigens, wie es nach den Untersuchungen ron Zaleski ${ }^{2}$ ) wahrscheinlich ist, in einer organischen Eisenverbindung zu suchen ist weiterhin geschieht, muss vorläufig noch unentschieden bleiben. $\mathrm{Ob}$ eine vermehrte Eisenausscheidung im Harne nach der Arsenwasserstoffvergiftung stattfindet, ist nicht leicht zu bestimmen, da der Harn fast immer hämoglobinhaltig ist. In dem enteiweissten Harne fand Baserin keinen gesteigerten Eisengehalt, doch ist es nicht auszuschliessen, dass bei dem Ausfällen des Hämoglobins auch noch andere Eisenverbindungen mitgerissen werden. - Ein dritter Weg für die Ausscheidung des bei der Gallenfarbstoffbildung abgespaltenen Eisens ist vielleicht in der Darmschleimhaut gegeben. Möglicherweise spielen hierbei auch die mit den unlöslichen eisenhaltigen Körnchen beladenen weissen Blutkörperchen eine gewisse Rolle. ${ }^{3}$ ) Schliesslich wäre es auch denkbar, dass das abgespaltene Eisen zunächst in der Leber liegen bleibt, um wieder assimilirt und zur Regeneration des Hämoglobins verwendet zu werden.

1) Vgl. Naunyn und Minkowski l. c. S. 25-26.

2) Studien über die Leber. I. Eisengehalt der Leber. Zeitschrift f. physiol. Chemie. X. Bd. S. 453-502. 1886.

3) Vgl. Siebel, Ueber das Schicksal von Fremdlrörpern in der Blutbahn. Virchow's Archiv. 54. Bd. S. 514. 1886. 


\section{Ueber die Umwandlung von Hypoxanthin in Harnsäure im Organismus der Vögel von W. v. Mach. ${ }^{1}$ )}

Bei seinen Untersuchungen über den Einfluss der Leberexstirpation auf den Stoffwechsel ${ }^{2}$ ) ist Referent zu der Annahme geführt worden, dass im Organismus der Vögel der bei Weitem grösste Theil der Harnsäure durch eine Synthese aus Ammoniak in der Leber gebildet werde, dass aber wahrscheinlich auch ausserhalb der Leber noch Harnsäure gebildet werden kann, und zwar möglicherweise durch eine einfache Oxydation der Xanthinkörper. Diese letztere Möglichkeit suchte nun Herr v. Mach experimentell zu prüfen, indem er Fütterungsversuche mit Hypoxanthin an Hühnern anstellte.

Ueber das Schicksal des Hypoxanthins im Stoffwechsel ist bis jetzt sehr wenig bekannt. Jaffé hat nach einer Mittheilung bei Ebstein ${ }^{3}$ ) Hunde mit Hypoxantlin gefiuttert, ohne zu einem positiven Resultate zu gelangen. Er fand nur, dass das eingegebene Hypoxanthin nicht unverändert im Harne ausgeschieden wurde. Anch Baginsky ${ }^{4}$ ) hat bei einem Hunde nach Eingabe von Hypoxanthin nur das Verschwinden dieser Substanz im Organismus constatiren können. Derselbe hielt die Möglichkeit nicht für ausgeschlossen, dass eine Umwandlung des Hypoxanthins in Harnsäure dabei stattgefunden hatte.

Die Resultate der v. Mach'schen Versuche sind aus Tabelle I und II auf Seite 149 ersichtlich.

In dem ersten Versuche wurde das Hypoxanthin in zweistuindlichen Dosen von $0,2 \mathrm{~g}$ eingegeben; bei dem zweiten Versuche wurden stündlich $0,2 \mathrm{~g}$ Hypoxanthin eingefübrt. Hieraus erklärt es sich wohl, dass die vermehrte Harnsäureausscheidung sich in dem ersten Versuche anf zwei Tage vertheilte, und dass in dem zweiten Versuche die Steigerung der Harnsäureausscheidung etwas geringer ausfiel, als nach der eingegebenen Menge des Hypoxanthins zu erwarten war.

Es kann nach diesen beiden Versuchen nicht zweifelhaft sein, dass eine Umwandlung des Hypoxanthins in Harnsäure im Organismus der Vögel stattfindet. Es darf daher auch mit grosser Wahrscheinlichkeit angenommen werden, dass im normalen Stoffwechsel der Vögel ein kleiner Theil der Harnsäure nicht

1) Die ausführlichere Mittheilung erfolgt in einer Inaug.-Dissertation.

2) Dieses Archiv. XXI. Bd. S. 86 .

3) Natur und Behandlung der Gicht. Wiesbaden 1882. S. 98.

4) Zeitschrift für physiologische Chemie. VIII. Bd. S. 397. 1884. 
Untersuchungen aus dem Laboratorium der med. Klinik zu Königsberg i. Pr. 149

TABBELLE I.

\begin{tabular}{|c|c|c|c|c|c|c|}
\hline Datum & $\begin{array}{c}\text { Gewioht } \\
\text { der } \\
\text { Excremente } \\
\text { in } \mathrm{g} \\
\end{array}$ & $\begin{array}{c}\text { Gesammte } \\
\text { stickstoff } \\
\text { in } \mathrm{g}\end{array}$ & $\begin{array}{l}\text { Harn- } \\
\text { säure } \\
\text { in } g\end{array}$ & $\begin{array}{l}\text { Körper- } \\
\text { gewieht } \\
\text { in } g\end{array}$ & Nabrung & Bemerkungen \\
\hline $15 . / \mathrm{V} .86$ & 35,7 & 0,8407 & - & 1880 & $\begin{array}{l}45 \mathrm{~g} \text { Gerste } \\
50 \mathrm{~g} \mathrm{H}_{2} \mathrm{O}\end{array}$ & 一 \\
\hline 16. V. 86 & 47,6 & 0,8610 & 1,218 & 1870 & $=$ & 一 \\
\hline $17 . / \mathrm{V} .86$ & 35,2 & 0,8633 & 1,216 & 1870 & $=$ & - \\
\hline $18 . / \mathrm{V}, 86$ & 35,7 & 0,8526 & 1,342 & 1860 & $=$ & - \\
\hline 19./V. 86 & 47,1 & 1,3645 & 2,049 & 1850 & $=$ & $\begin{array}{c}1 \mathrm{~g} \text { Hypoxanthin } \\
\text { entspreohend } \\
0,4118 \mathrm{~N}\end{array}$ \\
\hline $20 . /$ V. 86 & 45,1 & 1,0060 & 1,601 & 1860 & $=$ & + \\
\hline 21./V. 86 & 33,1 & 0,6706 & 1,180 & 1840 & $=$ & - \\
\hline $22 . / V .86$ & 34,1 & 0,7695 & - & 1860 & $=$ & - \\
\hline
\end{tabular}

TABeLLE II.

\begin{tabular}{|c|c|c|c|c|c|c|}
\hline Datum & $\begin{array}{c}\text { Gewicht } \\
\text { der } \\
\text { Excremente } \\
\text { in } g \\
\end{array}$ & $\begin{array}{c}\text { Gesammt- } \\
\text { stickstoff } \\
\text { in } g\end{array}$ & $\begin{array}{c}\text { Harn- } \\
\text { säure } \\
\text { in g }\end{array}$ & $\begin{array}{l}\text { Körper- } \\
\text { gewicht } \\
\text { in } g\end{array}$ & Nahrung & Bemerkungen \\
\hline 1. $/$ XI. 86 & 44,0 & 0,6270 & 0,7726 & 1795 & $\begin{array}{l}40 \mathrm{~g} \text { Gerste } \\
50 \mathrm{~g} \mathrm{H}_{2} \mathrm{O}\end{array}$ & 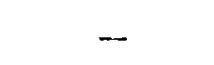 \\
\hline 2./X1. 86 & 37,4 & 0,6237 & $\overline{0-0}$ & 1785 & - & - \\
\hline 3./XI. 86 & 38,9 & 0,5532 & 0,7858 & 1760 & - & - \\
\hline 4./XI. 86 & 34,6 & 0,5154 & 0,7808 & 1750 & - & 19 \\
\hline 5. XI. 86 & 36,3 & 0,9511 & 1,5372 & 1750 & - & $\begin{array}{c}\text { entsprechend } \\
0,4941 \mathrm{~N}\end{array}$ \\
\hline 6./XI. 86 & 37,4 & 0,5563 & 0,8254 & 1740 & 一 & $\frac{0,7012}{-}$ \\
\hline 7./XI. 86 & 41,0 & 0,5291 & 0,7667 & 1730 & - & - \\
\hline 8./XI. 86 & 34,9 & 0,5436 & 0,7144 & 1725 & - & - \\
\hline
\end{tabular}

durch eine Synthese aus Ammoniak, sondern durch Oxydation der Xanthinkörper entsteht, welche als Spaltungsproducte der Nucleine (Kossel $\left.{ }^{1}\right)$ ) im Thierkörper auftreten. Weitere Versuche sollen ergeben, ob diese Art der Harnsäurebildung auch nach Ausschaltung der Leber noch möglich ist, und ob auch im Organismus der Säugethiere und speciell des Menschen, wie man es längst vermuthet hat, das Hypoxanthin als eine Vorstufe der Harnsäure zu betrachten ist. Es ist ohne Weiteres klar, dass gerade für den Organismus der Säugethiere diese Entstehungsweise der Harnsäure von besonderem Interesse ist, da hier durch Synthese aus Ammoniak nicht Harnsäure, sondern Harnstoff entsteht.

1) Zeitschrift für physiologische Chemie. III. Bd. 